\title{
A patient with recurrent epistaxis (2010: 11a)
}

(C) European Society of Radiology 2010

\section{Case report}

An 88-year-old woman with a 5-year history of recurrent left epistaxis was reviewed in the Ear, Nose and Throat clinic. Endoscopy showed a large mass occupying the post-nasal space. Computed tomography and magnetic resonance imaging (Fig. 1) were performed. Similar appearances had been demonstrated on a previous CT study obtained 5 years earlier with only minor interval progression compared with the current imaging.

\section{What is the diagnosis?}

Readers are invited to supply one possible diagnosis via electronic means to: robert.hermans@uzleuven.be
The subject of the e-mail should include 'Interpretation Corner' and the number given above (e.g., Interpretation Corner 2009: 1a). You should include your name, title, address, fax and phone number.

Deadline: one clear calendar month from the distribution date.

Three months after the initial publication of the case history, the authors will publish the final diagnosis and a brief summary. The summary will describe exactly how the case was investigated at the host institution, how the diagnosis was established and the teaching points of the case in question.

The names of the first 25 radiologists submitting the correct diagnosis will be published (only one from any individual centre and none from the host institution!).

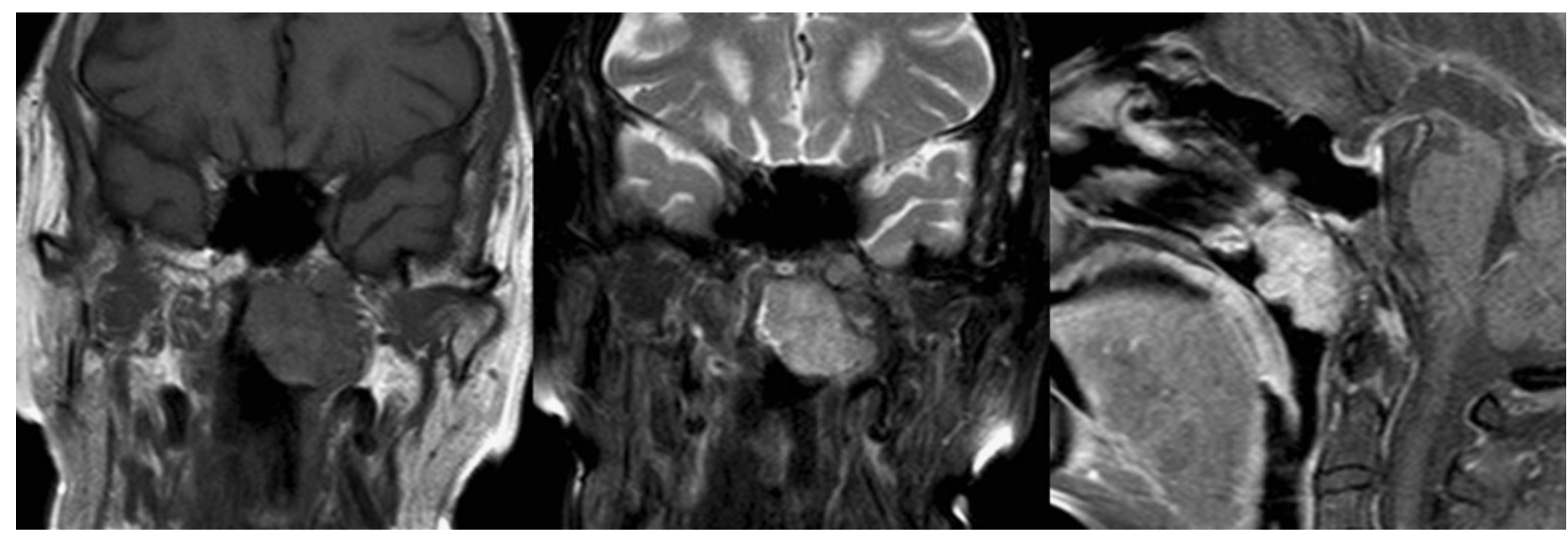

Fig. 1 Coronal T1, SPAIR and sagittal T1-weighted post-gadolinium MR images 